$$
\sum_{n=1}^{\infty} a_{m n} x_{n}, \quad \quad\left(m=m_{1}, m_{2}, \cdots\right)
$$

converges. This however cannot occur if $\mathfrak{A}$ is regular in the sense of the definition above, according to which all series

$$
\sum_{n=1}^{\infty} a_{m n} x_{n}, \quad\left(m \geqq m^{\prime}(x)\right),
$$

must converge.

The existence of a fixed $m_{0}$ is thus established, and at the same time it is shown that our modified definition of regularity of $\mathfrak{A}$ is equivalent to the classical one. It is clear that analogous considerations can be applied when instead of summation of series we deal with summation of integrals.

Brown UnIVERSITY

\title{
AN EXTENSION TO POLYGAMMA FUNCTIONS OF A THEOREM OF GAUSS*
}

BY H. T. DAVIS

1. Introduction. By the polygamma functions we mean the derivatives of $\log \Gamma(x)$, that is, $\Psi(x)=\Gamma^{\prime}(x) / \Gamma(x), \Psi^{\prime}(x), \cdots$, $\Psi^{(n)}(x) . \dagger$ These functions satisfy the difference equations:

$$
\Psi^{(n)}(x+1)-\Psi^{(n)}(x)=(-1)^{n} \frac{n !}{x^{n+1}},
$$

$$
\Psi^{(n)}(1-x)+(-1)^{n+1} \Psi^{(n)}(x)=A_{n}(x), A_{n}(x)=\frac{d^{n}}{d x^{n}}(\pi \operatorname{ctn} \pi x)
$$

subject to the boundary condition,

$$
\Psi^{(n)}(1)=(-1)^{n+1} n ! S_{n+1},
$$

where we employ the abbreviation

$$
S_{m}=1+1 / 2^{m}+1 / 3^{m}+\cdots \cdot
$$

* Presented to the Society, December 27, 1934.

† The name polygamma is suggested by the paper, Tables of the Digamma and Trigamma Functions, by Eleanor Pairman, Tracts for Computers, No. 1, 1919. 
One of the most striking identities satisfied by these functions is the following:

$$
\begin{aligned}
\Psi^{(m)}(n x)= & \left(1 / n^{m+1}\right)\left[\Psi^{(m)}(x)+\Psi^{(m)}(x+1 / n)\right. \\
& \left.+\cdots+\Psi^{(m)}(x+1-1 / n)\right]
\end{aligned}
$$

which holds for $m>0$. When $m=0$ the right member must be augmented by $\log n$.

In the construction of his celebrated tables of $\Psi(x)$ to 18 decimal places, Gauss* did not avail himself of the asymptotic expansion of this function, but employed rather a singularly elegant theorem by means of which values of $\Psi(x)$ for rational values of the argument could be explicitly determined. Although most of the theorems and identities associated with the polygamma functions can be derived by the simple device of differentiating the corresponding identities associated with $\Psi(x)$, this method is not possible for the arithmetical theorem of Gauss.

It is the purpose of this note, therefore, to develop the analogous expansions for $\Psi^{(n)}(p / q)$, where $p$ and $q$ are integers, $p<q$.

2. The Theorem. We shall prove the following theorem.

If $p$ and $q$ are integers, $p<q$, and if $n$ is greater than 0 , then the following formulas hold:

$$
\begin{aligned}
& \Psi^{(n)}(p / q)+\Psi^{(n)}(1-p / q) \\
& \quad=(-1)^{n+1} 2 \cdot n ! q^{n} \sum_{r=1}^{q-1} \cos (2 \pi r p / q) L^{(n+1)}(2 \pi r / q)+2 q^{n} \Psi^{(n)}(1) \\
& \Psi^{(n)}(p / q)-\Psi^{(n)}(1-p / q) \\
& \quad=(-1)^{n+1} 2 \cdot n ! q^{n} \sum_{r=1}^{q-1} \sin (2 \pi r p / q) M^{(n+1)}(2 \pi r / q)
\end{aligned}
$$

where we employ the abbreviations

$$
L^{(m)}(x)=\sum_{n=1}^{\infty} \cos n x / n^{m}, \quad M^{(m)}(x)=\sum_{n=1}^{\infty} \sin n x / n^{m} .
$$

Proof. If in the definitive expansion 162.

* Disquisitiones Generales Circa Seriem Infinitam, Werke, vol. 3, pp. 125- 


$$
\Psi^{(n)}(x)=(-1)^{n+1} n ! \sum_{k=0}^{\infty} 1 /(x+k)^{n+1}, \quad(n>0),
$$

we replace $x$ by $r / q$, multiply each $\Psi^{(n)}(r / q)$ by $\cos (2 m \pi r / q)$, and form the $\operatorname{sum} \sum_{r=1}^{q} \cos (2 m \pi r / q) \Psi^{(n)}(r / q)$, we shall have

$$
\begin{aligned}
& \sum_{r=1}^{q} \cos (2 m \pi r / q) \Psi^{(n)}(r / q) \\
& =(-1)^{n+1} n ! \sum_{k=0}^{\infty} \sum_{r=1}^{q} \cos (2 m \pi r / q) q^{n+1} /(r+q k)^{n+1} .
\end{aligned}
$$

But if we note that $\cos [2 m \pi(s q+r) / q]=\cos (2 m \pi r / q)$, then this sum takes the simple form

$$
\begin{aligned}
\sum_{r=1}^{q} \cos (2 m \pi r / q) \Psi^{(n)}(r / q) & =(-1)^{n+1} n ! q^{n+1} \sum_{r=1}^{\infty} \cos (2 m \pi r / q) / r^{n+1} \\
& =(-1)^{n+1} n ! q^{n+1} L^{(n+1)}(2 m \pi / q) .
\end{aligned}
$$

If now we let $m$ be successively $1,2,3, \cdots, q-1$ and note that $\cos (2 m \pi) \Psi^{(n)}(1)=(-1)^{n+1} n ! S_{n+1}$, we obtain the following system of equations:

$$
\begin{aligned}
& \sum_{r=1}^{q-1} \cos (2 m \pi r / q) \Psi^{(n)}(r / q)=(-1)^{n+1} n ! q^{n+1} L^{(n+1)}(2 m \pi / q) \\
& +(-1)^{n} n ! S_{n+1}, \quad(m=1,2, \cdots, q-1) .
\end{aligned}
$$

To this system we then add the equation

$$
\sum_{r=1}^{q-1} \cos (2 \pi r) \Psi^{(n)}(r / q)=\left(q^{n+1}-1\right)(-1)^{n+1} n ! S_{n+1},
$$

which is recognized as equation (2) in which the members of the right-hand side have been successively multiplied by $\cos 2 \pi$, $\cos 4 \pi$, etc.

We now multiply each equation of the system successively by $\cos (2 \pi p / q), \cos (4 \pi p / q), \cos (6 \pi p / q)$, etc., and add, taking note of the well known identity

$$
\begin{aligned}
I_{p r}(q)= & \sum_{n=1}^{q} \cos (2 \pi n p / q) \cos (2 \pi n r / q) \\
= & \left\{\begin{array}{l}
0 \text { if neither } p-r \text { nor } p+r \text { is divisible by } q, \\
q / 2 \text { if either } p-r \text { or } p+r \text { but not both is divisible by } q, \\
q \text { if both } p-r \text { and } p+r \text { are divisible by } q .
\end{array}\right.
\end{aligned}
$$


Hence when $q$ is odd, we obtain the identity

$$
\begin{aligned}
\Psi^{(n)}(r / q) & +\Psi^{(n)}(1-r / q) \\
& =(-1)^{n+1} n ! \sum_{m=1}^{q-1} \cos (2 \pi m r / q) q^{n} \cdot L^{(n+1)}(2 \pi m / q) \\
& +2(-1)^{n+1} q^{n} n ! S_{n+1} .
\end{aligned}
$$

But when $q$ is even, there is no difference in argument. In that case there will be a middle term $\Psi^{(n)}(r / q)=\Psi^{(n)}(1-r / q)$ $=\Psi^{(n)}(1 / 2)$. But since $I=q$, we shall have $(1 / 2) q \Psi^{(n)}(1 / 2)$ $+(1 / 2) q \Psi^{(n)}(1 / 2)=(1 / 2) q \Psi^{(n)}(r / q)+(1 / 2) q \Psi^{(n)}(1-r / q)$, which is the same as the result obtained when $n$ was odd.

In order to obtain the second identity stated in the theorem we now repeat the argument just given, merely replacing cosines by sines and $L^{(n)}(x)$ by $M^{(n)}(x)$ and noting the well known identity,

$J_{p r}(q)=\sum_{n=1}^{q} \sin (2 \pi n p / q) \sin (2 \pi n r / q)$

$$
=\left\{\begin{array}{l}
0 \text { if neither } p-r \text { nor } p+r \text { is divisible by } q, \text { or if both } \\
\quad \text { are divisible by } q, \\
q / 2 \text { if } p-r \text { but not } p+r \text { is divisible by } q, \\
-q / 2 \text { if } p+r \text { but not } p-r \text { is divisible by } q .
\end{array}\right.
$$

We then obtain

$$
\begin{aligned}
\Psi^{(n)}(r / q)- & \Psi^{(n)}(1-r / q) \\
& =(-1)^{n+1} n ! q^{n} \sum_{m=1}^{q-1} \sin (2 \pi m r / q) M^{(m+1)}(2 \pi m / q) .
\end{aligned}
$$

From these identities and known values of $L^{(n)}(x)$ and $M^{(n)}(x)$ it is possible to make explicit determinations of $\Psi^{(n)}(x)$ for rational values of the argument. We first note the formulas, ${ }^{*}$

$$
\begin{array}{r}
\int_{0}^{x} L^{(n)}(t) d t=M^{(n+1)}(x), \quad \int_{0}^{x} M^{(n)}(t) d t=-L^{(n+1)}(x)+S_{n+1} ; \\
L^{(1)}(x)=-\log \left(2 \sin \frac{x}{2}\right), M^{(1)}(x)=\frac{(\pi-x)}{2}, \\
\quad(0<x<2 \pi) ;
\end{array}
$$

* See T. J. I'a. Bromwich, Infinite Series, 2d ed., 1926, pp. 359-364. 


$$
\begin{aligned}
& L^{(2)}(x)= \frac{(x-\pi)^{2}}{4}-\frac{\pi^{2}}{12}, \\
& M^{(3)}(x)=\frac{1}{12}\left\{(x-\pi)^{3}-\pi^{2} x+\pi^{3}\right\}, \quad(0 \leqq x \leqq 2 \pi) ; \\
& L^{(4)}(x)=\frac{1}{48}\left\{2 \pi^{2}(x-\pi)^{2}-(x-\pi)^{4}-\frac{7 \pi^{4}}{15}\right\}, \quad(0 \leqq x \leqq 2 \pi) ; \\
& M^{(5)}(x)=\frac{1}{720}\left\{10 \pi^{2}(x-\pi)^{3}-3(x-\pi)^{5}-7 \pi^{4} x+7 \pi^{5}\right\},(0 \leqq x \leqq 2 \pi) .
\end{aligned}
$$

In the general case $L^{(m)}(x)$ for even exponents and $M^{(m)}(x)$ for odd exponents can be expressed in terms of Bernoulli polynomials as follows.

$$
\begin{aligned}
L^{(2 n)}(x) & =(-1)^{n+1} 2^{2 n-1} \pi^{2 n} B_{2 n}(x / 2 \pi) /(2 n) ! \\
M^{(2 n+1)}(x) & =(-1)^{n+1} 2^{2 n} \pi^{2 n+1} B_{2 n+1}(x / 2 \pi) /(2 n+1) ! .
\end{aligned}
$$

Applying the theorem to the cases $q=2,3$, and 4 , we are able to derive the following values:

$$
\begin{aligned}
& \Psi^{(n)}(1 / 2)=(-1)^{n+1} n !\left(2^{n+1}-1\right) S_{n+1}, \\
& \Psi^{(n)}(1 / 4)=(1 / 2)(-1)^{n+1} n ! 4^{n+1}\left[T_{n+1}+\left(1-1 / 2^{n+1}\right) S_{n+1}\right], \\
& \Psi^{(n)}(3 / 4)=(1 / 2)(-1)^{n+1} n ! 4^{n+1}\left[-T_{n+1}+\left(1-1 / 2^{n+1}\right) S_{n+1}\right], \\
& \Psi^{(n)}(1 / 3)=(1 / 2)(-1)^{n+1} n !\left[3^{n+1} \sigma_{n+1}+\left(3^{n+1}-1\right) S_{n+1}\right], \\
& \Psi^{(n)}(2 / 3)=(1 / 2)(-1)^{n+1} n !\left[-3^{n+1} \sigma_{n+1}+\left(3^{n+1}-1\right) S_{n+1}\right],
\end{aligned}
$$

where we employ the abbreviations

$$
\begin{aligned}
S_{r}= & \sum_{m=1}^{\infty} 1 / m^{r}, \quad T_{r}=\sum_{m=1}^{\infty}(-1)^{m+1} /(2 m-1)^{r} \\
\sigma_{r}= & 1-1 / 2^{r}+1 / 4^{r}-1 / 5^{r}+1 / 7^{r}-1 / 8^{r} \\
& +1 / 10^{r}-1 / 11^{r}+\cdots .
\end{aligned}
$$

INDIANA UNIVERSITY 